J. Lake Sci. (湖泊科学), $2006, \mathbf{1 8}(4): 319-326$

http:// www. jlakes. org. E-mail: jlakes@ niglas. ac.cn

(c) 2006 by Journal of Lake Sciences

\title{
太湖梅梁湾有色可溶性有机物的空间分布及光学行为"
}

\author{
张运林, 秦伯强, 杨龙元 \\ (中国科学院南京地理与湖泊研究所, 南京 210008)
}

\begin{abstract}
摘 要:2004 年 3 月对太湖梅梁湾有色可溶性有机物 (CDOM) 的吸收和苂光等光学行为进行研究, 并由此探讨了 CDOM 的空间分布. 结果表明, 溶解性有机碳 (DOC) 的浓度在 $10.48-19.72 \mathrm{mg} / \mathrm{L}$ 间变化, 其均值为 $13.20 \pm 2.79 \mathrm{mg} / \mathrm{L}$; CDOM

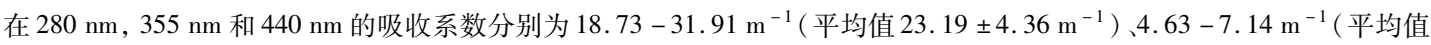
$5.76 \pm 0.91 \mathrm{~m}^{-1}$ ) 、1.45-2.99 m-1 (平均值 $1.92 \pm 0.40 \mathrm{~m}^{-1}$ ); $355 \mathrm{~nm}$ 波长处 CDOM 的比吸收系数为 $0.34-0.57 \mathrm{~L} /(\mathrm{mg}$ $\cdot \mathrm{m})$, 平均值 $0.44 \pm 0.06 \mathrm{~L} /(\mathrm{mg} \cdot \mathrm{m})$; 表征 $\mathrm{CDOM}$ 分子大小的比值 $a(250) / a(365)$ 变化范围为 $5.05-7.55 ; 355 \mathrm{~nm}$ 的激 发波长、 $450 \mathrm{~nm}$ 的发射波长处的荧光值的变化范围 $0.79-3.04 \mathrm{~nm}^{-1}$ (平均值 $1.69 \pm 0.77 \mathrm{~nm}^{-1}$ ). CDOM 吸收系数、DOC 浓度、苂光强度的分析显示 CDOM 浓度呈现从河口往湾内、湾口递减的趋势. CDOM 吸收与 DOC 浓度的相关性随波长的 降低而增加，在短波部分存在明显的正相关. $355 \mathrm{~nm}$ 处的荧光值、DOC 浓度与 CDOM 吸收系分别存在如下显著性正相关 关系: $F_{n}(355)=0.692( \pm 0.135) a(355)-2.297( \pm 0.786), a(355)=0.233( \pm 0.061) D O C+2.690( \pm 0.816) .280$ $-500 \mathrm{~nm} 、 280-360 \mathrm{~nm} 、 360-440 \mathrm{~nm}$ 指数函数斜率 $S$ 值分别为 $13.86 \pm 0.91 、 18.54 \pm 1.11 、 12.93 \pm 0.92 \mu \mathrm{m}^{-1}, S$ 值与比 吸收系数之间存在显著的负线性相关关系, 而与 $a(25) / a(365)$ 值则存在显著的正线性关系. 比吸收系数越大, $a(250) /$ $a(365)$ 值和 $S$ 值就越小, 对应的 CDOM 分子量就越大, 腐质酸的比例就越高.
\end{abstract}

关键词: 有色可溶性有机物 (CDOM) ; 苂光; 溶解性有机碳 (DOC) ; 指数函数斜率; 太湖

\section{Spatial distribution and absorption characteristics with relation to fluorescence of chromo- phoric dissolved organic matter (CDOM) in Meiliang Bay of Lake Taihu}

\section{ZHANG Yunlin, QIN Boqiang \& YANG Longyuan}

( Nanjing Institute of Geography \& Limnology, Chinese Academy of Sciences, Nanjing 210008,P. R. China)

\begin{abstract}
Chromophoric dissolved organic matter ( CDOM) is the light absorption fraction of dissolved organic carbon (DOC) that absorbs light in both the ultraviolet and visible ranges. The spatial and temporal distribution of CDOM in aquatic ecosystems can have important effects on ecosystem productivity, negatively impacting primary productivity due to CDOM absorbs light at both ultraviolet and visible wavelengths while positively impacting secondary productivity by fueling of microbial respiration by photo-degraded CDOM. In water color remote sensing, overlaps of pigment absorption spectra with CDOM absorption at blue wavelength generally complicate the use of chlorophyll a retrieval algorithms that are based on remotely sensed ocean color and lead to overestimated chlorophyll a concentration. In addition, high concentrations of CDOM can also act as a photoprotectant against UV damage for aquatic organisms. However, the protection against UV radiation provided by dissolved humic material for aquatic biota may be diminished if photodegradation of CDOM by UV radiation and acidification increase the UV transparency in lakes.
\end{abstract}

CDOM absorption measurements and their relationship with DOC, and fluorescence are presented in Meiliang Bay of Lake Taihu based on a field investigation and lab analysis to show the spatial distribution of CDOM. Absorption spectral of CDOM was measured from 240 to $800 \mathrm{~nm}$. Concentrations of DOC ranged from 10.48 to 19.72 mg/

* 中国科学院知识创新工程项目 (KZCX3 - SW - 334), 国家自然科学基金项目 (40573062,40501078) 联合资助. $2005-03-25$ 收稿; $2005-08-29$ 收修改稿. 张运林, 男, 1976 年生, 博士, 助理研究员; E-mail: ylzhang@ niglas. ac. cn. 
$\mathrm{L}$ with an average of $13.20 \pm 2.78 \mathrm{mg} / \mathrm{L}$. CDOM absorption coefficients at $280 \mathrm{~nm}$ and $355 \mathrm{~nm}$ were in the range $18.73-31.91 \mathrm{~m}^{-1}$ (average $23.19 \pm 4.36 \mathrm{~m}^{-1}$ ) and $4.63-7.14 \mathrm{~m}^{-1}$ (average $5.76 \pm 0.90 \mathrm{~m}^{-1}$ ), respectively. The values of the DOC-specific absorption coefficient at $355 \mathrm{~nm}$ ranged from 0.34 to $0.57 \mathrm{~L} /(\mathrm{mg} \cdot \mathrm{m})$. Fluorescence emission at $450 \mathrm{~nm}$, excited at $355 \mathrm{~nm}$, had a mean value of $1.69 \pm 0.77 \mathrm{~nm}^{-1}$. A significant lake zone difference is found in DOC concentration, CDOM absorption coefficient and fluorescence. This regional distribution pattern was in agreement with the location of sources of yellow substance: highest concentrations close to river mouth under the influence of river inflow, lower values in outlet of Meiliang bay. CDOM fluorescence and absorption coefficient were significantly and positively correlated. The results show a good correlation between CDOM absorption and DOC coefficients during $280-500 \mathrm{~nm}$ short wavelength intervals. The coefficient of variation between CDOM absorption and DOC concentration decreased with the increase of wavelength from 280 to $500 \mathrm{~nm}$. The linear regression equations between fluorescence, DOC concentration and absorption coefficients at $355 \mathrm{~nm}$ are: $F_{n}(355)$ $=0.692( \pm 0.135) a(355)-2.297( \pm 0.786), a(355)=0.233( \pm 0.061) D O C+2.690( \pm 0.816)$, respectively. The exponential slope coefficient ranged from 11.0 to $14.9 \mu \mathrm{m}^{-1}$ with a mean value $13.86 \pm 0.91 \mu \mathrm{m}^{-1}$, $15.8-20.7 \mu \mathrm{m}^{-1}$ with a mean value $18.54 \pm 1.11 \mu \mathrm{m}^{-1}$ and $9.9-13.9 \mu \mathrm{m}^{-1}$ with a mean value $12.93 \pm 0.92$ $\mu \mathrm{m}^{-1}$ over the $280-500 \mathrm{~nm}, 280-360 \mathrm{~nm}$ and $360-440 \mathrm{~nm}$ intervals. A significant negative linear correlation was found between spectral slope coefficient and DOC specific absorption coefficient, but a significant positive linear correlation for spectral slope coefficient and the ratio of $a(250) / a(365)$. Larger specific absorption coefficient corresponds to smaller $a(250) / a(365)$ and spectral slope coefficient, which shows higher ratio humic in CDOM. Keywords: Chromophoric dissolved organic matter ( CDOM); fluorescence; dissolved organic carbon (DOC); spectral slope coefficient; Lake Taihu

有色可溶性有机物 (Chromophoric dissolved organic matter: CDOM) ( 又称黄质, 在我国大多被称为黄色 物质) 存在于所有水体中, 它是溶解性有机物库的重要组成部分 ${ }^{[1]}$. 由腐质酸、富里酸、芳烃聚合物等一系 列物质组成, 主要是土壤和水生植物降解的产物 ${ }^{[2]}$. CDOM 的吸收光谱是对紫外光吸收最强, 到近红外光 趋向于零. 由于它对紫外辐射的强烈吸收, 因而限制了生物有害的 UV-B 辐射 $(280-320 \mathrm{~nm})$ 穿透深度, 保 护了水生生物, 对水体生态系统有明显影响 ${ }^{[3-6]}$. CDOM 的吸收还延展到可见光的蓝光部分, 与浮游植物 叶绿素 $a$ 和非藻类颗粒物的吸收重叠, 因而影响水体初级生产力、干扰了浮游植物生物量的水色遥 感 ${ }^{[7-9]}$. 由于 CDOM 化学组成极为复杂, 要想从水体中提取 CDOM 相当困难, 因而当前人们多从其吸收和 苂光等光学行为来开展对它的研究. 国际上对 CDOM 的吸收和苂光研究由来已久 ${ }^{[10-13]}$, 最近国内也开展 了部分相关研究, 在海洋里, 吴永森等 ${ }^{[14]}$ 对实验室配制的不同浓度黄色物质水溶液、实验室藻类培养液和 胶州湾水域黄色物质水样的光吸收特性进行了研究, 并探讨了指数函数斜率 $S$ 值的数值范围; 陈楚群 等 ${ }^{[15]}$ 在珠江口海域采用正演方法模拟水体光谱并以此反演水体中黄色物质浓度; 朱建华等 ${ }^{[16]}$ 对我国黄东 海域黄色物质的光谱吸收系数进行了探讨. 对于内陆水体, 杨顶田等 ${ }^{[17]}$ 对长江中下游湖泊的巢湖和龙感 湖作了一些对比性研究, 发现藻型湖泊 CDOM 的浓度明显高于草型湖泊, 这在太湖草、藻型湖区也得到进 一步验证 ${ }^{[18]}$; 张运林等 ${ }^{[19]}$ 就太湖梅梁湾 CDOM 吸收对紫外辐射衰减的贡献进行了一些探讨; 马荣华等 ${ }^{[20]}$ 从水色遥感的角度分析了东太湖其他水色因子对 CDOM 吸收光谱影响. 本文重点探讨梅梁湾 CDOM 空间 分布、吸收系数与 DOC 浓度、苂光强度的关系以及能表征 CDOM 组成的几个参数之间的内在关系, 以期深 人理解 CDOM 这一类复杂物质的光学行为及其地球化学行为.

\section{1 材料与方法}

\section{1 采样时间与站位}

2004 年 3 月 4 日在梅梁湾共布设了 16 个点进行样品的采集分析(图 1).

\subsection{CDOM 吸收和 DOC 浓度测定}

CDOM 的光谱吸收系数测定采用通过 GF/F 过滤的水样在 UV2401 分光光度计下测定其吸光度, 然后 
根据 $(1)$ 式进行计算得到各波长的吸收系数 ${ }^{[10]}$.

$$
a\left(\lambda^{\prime}\right)=2.303 D(\lambda) / r
$$

式中, $a\left(\lambda^{\prime}\right)$ 为波长 $\lambda$ 未校正的吸收系数 $\left(\mathrm{m}^{-1}\right), D$ $(\lambda)$ 为吸光度, $r$ 为光程路径 $(m)$.

由于过滤清液可能残留细小颗粒会引起散射, 为此需作如下散射效应订正 ${ }^{[21]}$ :

$$
a(\lambda)=a\left(\lambda^{\prime}\right)-a(750) \lambda / 750
$$

式中, $a(\lambda)$ 为波长 $\lambda$ 的吸收系数 $\left(\mathrm{m}^{-1}\right) ; a\left(\lambda^{\prime}\right)$ 为波 长 $\lambda$ 的未校正吸收系数 $\left(\mathrm{m}^{-1}\right) ; \lambda$ 波长 $(\mathrm{nm})$. 由于 CDOM 由氨基酸、糖、氨基糖、脂肪酸、类胡萝卜素、 氯纶色素、碳水化合物和酚等一系列物质组成,其浓 度无法测定，最常用方法是用 $355 \mathrm{~nm} 、 375 \mathrm{~nm} 、 440$

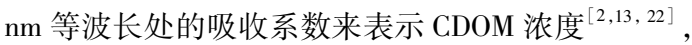

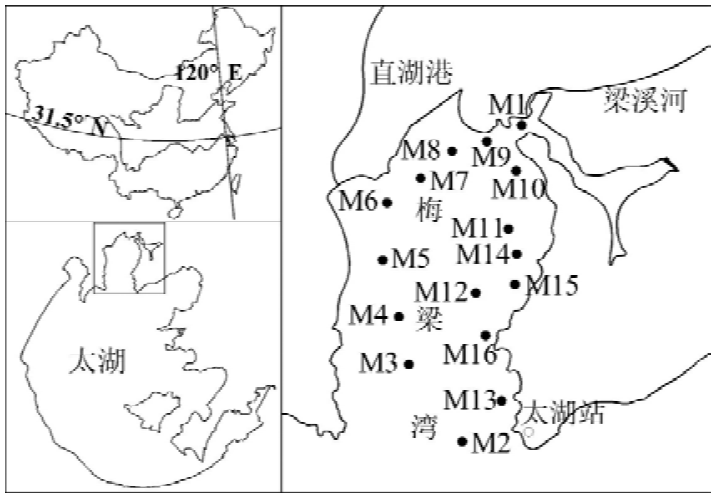

图 1 梅梁湾采样点位置图

Fig. 1 Location of sampling sites in Meiliang Bay 吸收系数越大对应的 CDOM 浓度就越高. 本文以 $355 \mathrm{~nm}$ 的吸收系数来表示 CDOM 浓度并以此波长处的吸收与 DOC 浓度及菼光值建立关系. 基于 $250 \mathrm{~nm}$ 和 $365 \mathrm{~nm}$ 波长 CDOM 吸收系数的比值来估算 CDOM 分子大小, 其比值越小分子量则越大 ${ }^{[6,23]}, \mathrm{CDOM}$ 分 子量的大小大致能反映腐质酸、富里酸在 $\mathrm{CDOM}$ 中的比例，因为腐质酸的分子量一般较大，而富里酸则较 小, 因而 CDOM 分子量越大, 腐质酸的比例就越高.

溶解性有机碳 DOC 的测定方法是用 Whatman GF/F 玻璃纤维滤膜进行过滤，将过滤清液在 1020 型 TOC 仪上测定, 仪器监测范围为 $0.5-500 \mathrm{mg} / \mathrm{L}$, 相对误差为 $\pm 3 \%$.

CDOM 的比吸收系数 $a^{*}(\lambda)$ 按 $(3)$ 式计算:

$$
a^{*}(\lambda)=a(\lambda) / \mathrm{DOC}
$$

式中, $a^{*}(\lambda)$ 为比吸收系数 $\mathrm{L} /(\mathrm{mg} \cdot \mathrm{m}) ; a(\lambda)$ 为波长 $\lambda$ 的吸收系数 $\left(\mathrm{m}^{-1}\right)$; DOC 为溶解性有机碳的浓度 $(\mathrm{mg} / \mathrm{L})$.

\section{3 荧光的测定}

CDOM 在短波紫外光的激发下会发出长于激发波长的苂光，苂光强度的高低能反映 CDOM 的浓度大 小，一般苂光强度越高 CDOM 浓度也就越高，本文选取国际上通用的 $355 \mathrm{~nm}$ 作为激发波长，450 nm 作为 发射波长, 用 Mill - Q 水做参比样, 在 RF5301 苂光光度计上测定其苂光值 ${ }^{[1]}$.

\section{4 指数函数斜率 $S$ 的计算}

CDOM 对 $500 \mathrm{~nm}$ 以下光谱吸收基本上呈现指数衰减的规律, Bricaud 等 $^{[10]}$ 提出如下的公式:

$$
a(\lambda)=a\left(\lambda_{0}\right) \exp \left[S\left(\lambda_{0}-\lambda\right)\right]
$$

式中, $a(\lambda)$ 是 CDOM 的吸收系数 $\left(\mathrm{m}^{-1}\right), \lambda$ 是波长 $(\mathrm{nm}), \lambda_{0}$ 是参照波长 $(\mathrm{nm})$ ，一般取 $440 \mathrm{~nm}, S$ 是指数函 数曲线斜率参数, 可以拟合得到不同波段的 $S$ 值.

\section{5 数据分析}

运用 SPSS11.0 进行数据统计分析, 包括计算平均值、标准差、线性及非线性拟合等.

\section{2 结果与讨论}

\section{1 CDOM 的光谱吸收和空间分布}

图 2 给出梅梁湾典型站位 CDOM 的吸收光谱, 其吸收曲线表现出高度的一致性, 在 $700 \mathrm{~nm}$ 附近的红 外波段趋向于零，在 $280-320 \mathrm{~nm}$ 紫光外波段最大，吸收系数自然对数值随波长变化基本上表现为直线. 在 $280 \mathrm{~nm}, 355 \mathrm{~nm}$ 和 $440 \mathrm{~nm}$ 的吸收系数变化范围分别为 $18.73-31.91 \mathrm{~m}^{-1}$ (平均值 $23.19 \pm 4.36 \mathrm{~m}^{-1}$ ) 、 $4.63-7.14 \mathrm{~m}^{-1}$ (平均值 $5.76 \pm 0.91 \mathrm{~m}^{-1}$ ) 、1.45-2.99 $\mathrm{m}^{-1}$ (平均值 $1.92 \pm 0.40 \mathrm{~m}^{-1}$ ) (表 1 ). 相对太湖不 同湖区间 ${ }^{[18]}$,梅梁湾内 CDOM 吸收系数差异不是特别大, 表征 CDOM 浓度的 $a(355)$ 均在 $4 \mathrm{~m}^{-1}$ 以上, 总体 上呈现河口和湾内各点 CDOM 浓度一般还是要高于湾口各点, 如 M1、M7 - M10 的 $a(355)$ 均要高于 $6 \mathrm{~m}^{-1}$, 
表 1 特征波段吸收系数、比吸收系数、指数函数斜率、DOC 浓度及苂光

Tab. 1 Absorption coefficients, DOC-specific absorption coefficients, expotential slope coefficient,

DOC concentrations and fluorescence

\begin{tabular}{|c|c|c|c|c|c|c|c|c|c|c|c|c|}
\hline \multirow{2}{*}{ 样点 } & \multicolumn{3}{|c|}{$(\lambda)\left(m^{-1}\right)$} & \multirow{2}{*}{$\begin{array}{l}-a(250)- \\
/ a(365)\end{array}$} & \multicolumn{3}{|c|}{$a^{*}(\lambda)(\mathrm{L} /(\mathrm{mg} \cdot \mathrm{m}))$} & \multicolumn{3}{|c|}{$S\left(\mu \mathrm{m}^{-1}\right)$} & \multirow{2}{*}{\multicolumn{2}{|c|}{$\begin{array}{c}\text { DOC } F_{n}(355) \\
(\mathrm{mg} / \mathrm{L})\left(\mathrm{nm}^{-1}\right)\end{array}$}} \\
\hline & 280 & 5 & 40 & & 280 & 355 & 440 & $S_{1}$ & $S_{2}$ & $S_{3}$ & & \\
\hline $\mathrm{M}^{\#}$ & 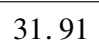 & 1 & re & & . & & & 17. & 20.7 & & & 3.04 \\
\hline $\mathrm{M} 2^{\#}$ & 20.95 & 3.1 & 03 & & 2.00 & 5 & 19 & 9 & 17.2 & 2 & 49 & 79 \\
\hline $\mathrm{M}^{\#}$ & .25 & . & . & 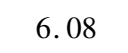 & .00 & 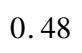 & xe & 13.4 & 等 & 12.7 & 1180 & 1.14 \\
\hline $\mathrm{M} 4^{\#}$ & 19.10 & 4.9 & 61 & & 72 & 5 & 15 & 13.9 & 18. & 13 & 07 & 0.92 \\
\hline $\mathrm{M}^{\#}$ & 10.70 & 4 & (נד & & OJ & & & 17.4 & 18.7 & 3 & & 0.90 \\
\hline $\mathrm{M}^{\#}$ & 19.19 & 4.7 & 1.47 & 1 & 1.77 & 44 & 14 & 14.6 & 18.7 & 13.8 & 10.81 & 1.02 \\
\hline $\mathrm{M} 7^{\#}$ & 28.12 & 1.14 & 2.50 & & 1.01 & & & 15.7 & 0.7 & & 03 & 3.02 \\
\hline $\mathrm{M} 8^{\#}$ & 25.35 & 0.2 & 2.05 & & 1.86 & . & (1) & 14.0 & 18.6 & 13 & 13.60 & 1.99 \\
\hline M9 ${ }^{\#}$ & 29.04 & & & & & & & & & & & 2.74 \\
\hline M10 & 29.00 & 6.9 & 6 & s & 1.74 & 0 & 0.1 & 13.9 & 19.0 & 13.0 & 16 & 2.61 \\
\hline M11 ${ }^{\#}$ & 5.46 & 5.85 & 1.80 & & 1.01 & & & 14.7 & 19.0 & 13.6 & 15.77 & 1.93 \\
\hline M12 ${ }^{\#}$ & 19.58 & 4.95 & 1.62 & & 1.75 & 0.44 & 0.15 & 13.9 & 18.4 & 12.9 & 11.19 & 1.31 \\
\hline M13 & 18.73 & 4.70 & 1.55 & 0.51 & 1.19 & $0.4 J$ & 0.15 & 15.9 & 10.4 & 12.9 & 10.40 & 1.22 \\
\hline M14 & 21.49 & 5.23 & 1.71 & 6.64 & 1.56 & 0.38 & 0.12 & 14.0 & 18.9 & 13.0 & 13.80 & 1.43 \\
\hline M15 & 19.97 & 5.07 & $1.6 /$ & 0.52 & 1.86 & 0.47 & 0.10 & 14.0 & 18.4 & 12.9 & 10.74 & 1.39 \\
\hline M16 & 23.16 & 7.03 & 2.99 & 5.05 & 1.89 & 0.57 & 0.24 & 11.0 & 15.8 & 9.9 & 12.25 & 1.57 \\
\hline
\end{tabular}

1) $S_{1}: 280-500 \mathrm{~nm} ; S_{2}: 280-360 \mathrm{~nm} ; S_{3}: 360-440 \mathrm{~nm}$.
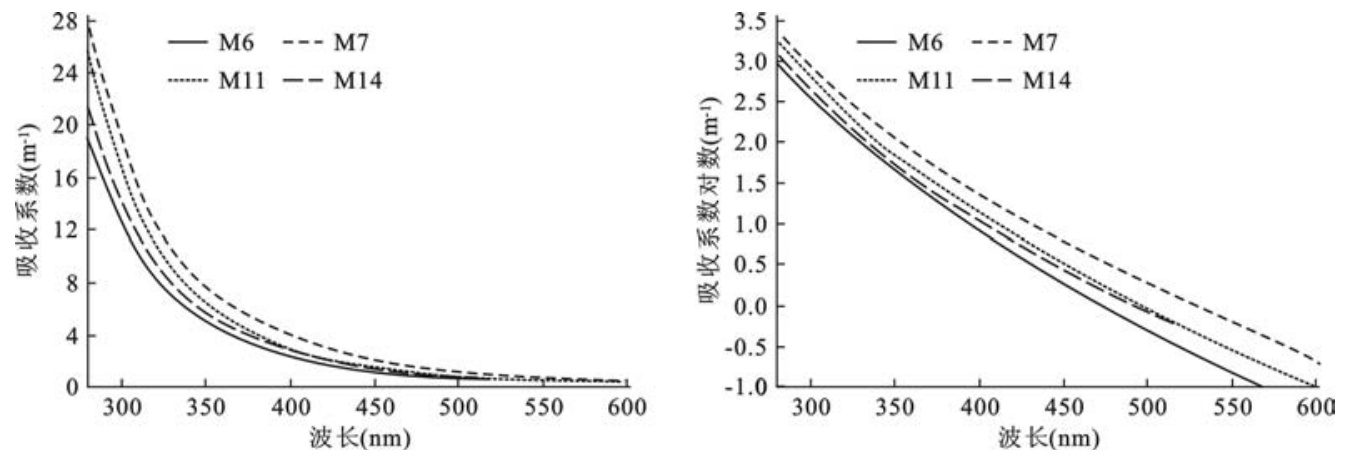

图 2 典型站位 CDOM 吸收系数光谱分布

Fig. 2 Absorption coefficient spectra of CDOM at typical sampling sites

最高值为 $7.14 \mathrm{~m}^{-1}$, 出现在 M7 点, 而其他各点除了 M16 外均要低于 $6 \mathrm{~m}^{-1}$ 。从 $280 \mathrm{~nm}$ 波长处 CDOM 吸收 系数来看, 其最高值为 $31.91 \mathrm{~m}^{-1}$, 位于梁溪河人湖口的 M1\#点. 由此大致上可以看出, 梅梁湾内 CDOM 浓 度呈现从河口往湾内、湾口递减的趋势, 这与 2003 年 12 月和 2004 年 4 月在梅梁湾观测到的结果大致相 似 ${ }^{[18,19]}$, 反映了冬、春季浮游植物浓度较低时, 梅梁湾 CDOM 主要受陆源河流的影响, 但到夏季浮游植物 大量生长后, 其腐烂降解的产物也将对水体中 CDOM 浓度产生重要影响. 梅梁湾内 CDOM 浓度的这种空 
间分布与许多海湾中的研究结果也相似 ${ }^{[1,11]}$, 体现了河流陆源对 CDOM 的重要贡献.

太湖梅梁湾 CDOM 吸收系数与 Frenette 等 ${ }^{[24]}$ 在河相的大型浅水湖泊 Saint - Pierre 湖的研究结果比较 接近, 并且都落在 Kirk $^{[2]}$ 报道的吸收系数范围之内, 但比 Morris 等 ${ }^{[25]}$ 在美国东北部及科罗拉多州、阿拉斯 加州的湖泊调查结果要略高, 并明显高于沿岸带和海洋中的值 ${ }^{[2,12]}$. 由于 CDOM 在紫外和蓝光波段吸收 最强, 因而许多研究也常常借助 CDOM 的吸收系数来反映 UV-B 辐射在水体的衰减情况, 建立了漫射衰减 系数与 CDOM 吸收系数的线性回归关系 ${ }^{[5,25]}$. 表征 CDOM 分子大小比值 $a(25) / a(365)$ 的变化范围为 $5.05-7.55$, 数值上与国际上同类研究结果比较一致 ${ }^{[6,23]}$. 太湖梅梁湾 CDOM 浓度较高, 一方面由于其大 量吸收 UV-B 辐射将降低 UV-B 对湖泊生物损伤, 但另一方面由于 CDOM 吸收 UV-B 后会快速发生光降解 (Photodegradation), UV-B“光漂白” (Photobleaching) 致使湖泊水色变浅, CDOM 浓度降低, UV-B 辐射影响 深度增加，而长期处于高 CDOM 保护下的生物由于缺乏相应的光保护和修复机制此时更易受到 UV-B 的 损伤. 此外, CDOM 光化学降解还会产生一些毒性物质, 如对细菌有害的单重态氧、羟基和超氧自由基. 因 而关于湖泊内陆水体中 CDOM 生物地球化学及光化学降解的研究应引起高度重视.

\subsection{DOC 及与 CDOM 吸收的关系}

此次调查 DOC 的浓度在 $10.48-19.72 \mathrm{mg} / \mathrm{L}$ 间变化, 其均值为 $13.20 \pm 2.79 \mathrm{mg} / \mathrm{L}$, 最高值出现在梁 溪河口的 M1 ${ }^{\#}$ 点, 最低值出现在位于湾口附近的 M2、M13 点, DOC 空间分布大致呈现从湾内向湾口递减的 趋势, 这与前面分析的 CDOM 浓度空间分布也较为一致. 梅梁湾 DOC 浓度与国际上同类湖泊报道的结果 比较接近, 如 Huovinen 等 ${ }^{[6]}$ 曾报道芬兰中部 5 个高腐殖质湖泊 DOC 浓度在 $4.9-14.9 \mathrm{mg} / \mathrm{L}$ 之间, 由于 CDOM 是溶解性碳库的重要组成部分, 已有的许多研究都显示 DOC 浓度与 CDOM 吸收之间存在显著性正 相关, 并可以借助这些相关模式来模拟 DOC 浓度的变化 ${ }^{[1,11]} \cdot a(280), a(355)$ 与 DOC 浓度均存在显著性 正相关, 关系式如下: $a(280)=1.484( \pm 0.133) D O C+3.602( \pm 1.795)\left(R^{2}=0.90, N=16, P \leqslant\right.$ $0.0001) ; a(355)=0.233( \pm 0.061) D O C+2.690( \pm 0.816)\left(R^{2}=0.51, N=16, P \leqslant 0.005\right)$, 并且一般随 波长的降低, 两者相关性越来越好, 这在其他的研究中也得到证实 ${ }^{[18,19,26]}$.

CDOM 比吸收系数可以作为区分 CDOM 来源和类型的参数. $355 \mathrm{~nm}$ 波长处的比吸收系数为 $0.34-$ $0.57 \mathrm{~L} /(\mathrm{mg} \cdot \mathrm{m})$, 平均值 $0.44 \pm 0.06 \mathrm{~L} /(\mathrm{mg} \cdot \mathrm{m})$, 与 Morris 等 ${ }^{[25]}$ 报道的美国东北部及科罗拉多州、阿拉 斯加州的湖泊中的值较为一致, 但一般都大于海湾和沿岸带报道的结果, 明显低于 Seritti 等 ${ }^{[11]}$ 报道的河 流中的值, 反映了不同类型生态系统 CDOM 来源和类型的差异. 关于 CDOM 比吸收系数的变化不同研究

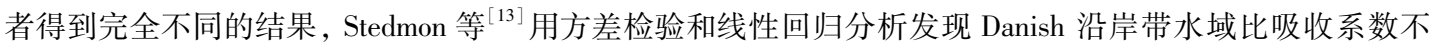
存在显著性变化, 可以用平均值代替整个区域. Gallie ${ }^{[27]}$ 的研究发现 CDOM 比吸收系数与湖泊 $\mathrm{pH}$ 值、DOC 浓度存在显著的正相关. 然而 Yacobi 等 ${ }^{[28]}$ 却发现 $440 \mathrm{~nm}$ 波长处比吸收系数与 DOC 浓度存在负相关关系, 本研究也证实了 CDOM 比吸收系数与 DOC 浓度大致存在负的线性相关关系, 相关性决定系数在 0.3 左 右，显著性水平小于 0.05 .

此外, Yacobi 等 ${ }^{[28]}$ 还提出高的比吸收系数 $a^{*}(440)$ 一般对应着高比例的大 CDOM 分子, 由于 $a$ $(250) / a(365)$ 值能表征 CDOM 分子的大小, 为此对 $a^{*}$ (355) 与 $a(250) / a(365)$ 值进行线性相关分析, 发 现两者存在非常显著的负相关, 相关式为: $a^{*}(355)=-0.1003 a(250) / a(365)+1.1008\left(R^{2}=0.86, N=\right.$ $16, P \leqslant 0.0001)$. 由于 $a(250) / a(365)$ 值越小分子量则越大, 而 $a(250) / a(365)$ 值越小, 对应的 $a^{*}(355)$ 也就越大, 因而证实了 Yacobi 等人的观点, 说明借助比吸收系数也可以来反映 CDOM 的分子大小.

\section{3 指数函数斜率 $S$ 值}

表 1 给出了 $280-500 \mathrm{~nm} 、 280-360 \mathrm{~nm} 、 360-440 \mathrm{~nm} 3$ 个波段基于最小二乘法得到的指数函数斜率值, 3 个波段 16 个采样点的平均值分别为 $13.86 \pm 0.91 、 18.54 \pm 1.11 、 12.93 \pm 0.92 \mu^{-1}$. 同一波段内各点 $S$ 值差异很小，但不同的波段间 $S$ 值存在一定差异，一般短波波段值要高于长波波段，如 $280-360 \mathrm{~nm}$ 的 $S 2$ 均值是 360-440 nm 的 $S 3$ 均值的 1.43 倍, 差异非常明显, 说明 CDOM 的吸收越到短波增加越明显, 这也 反映了 $S$ 值与 $\mathrm{CDOM}$ 浓度无关, 但与波长的选择及 $\mathrm{CDOM}$ 物质组成有关. 从 3 波段指数拟合得到的 $S$ 值 来看, 都很好的落于 Markager 等 ${ }^{[29]}$ 报道的淡水湖中 $10-25 \mu \mathrm{m}^{-1}$ 范围内, 并且也一般高于沿岸带和海洋 中的研究结果, 进一步验证了内陆淡水湖泊中 $S$ 值比海洋中略高的结论. 同一波段内 $S$ 值的差异可能主要 
来自于 CDOM 的化学组成, 既腐质酸和富里酸的比例, Carder 等 ${ }^{[7]}$ 曾发现来自密西西比河浑水里富含富 里酸的海生样品其 $S$ 值 $\left(S=19.4 \mu^{-1}\right)$ 明显要高于来自于墨西哥湾富含腐质酸的海生样品 $(S=10.0$ $\mu \mathrm{m}^{-1}$ ). 由于 $S$ 值能反映 CDOM 的组成和来源, 因而大多数研究者对其颇为关注, 关于 $S$ 值与各参数的相 关性有不同报道. Yacobi 等 ${ }^{[28]}$ 发现 $440 \mathrm{~nm}$ 波长处比吸收系数 $a^{*}$ (440) 与 $S$ 值存在明显的负相关, 而 Del Castillo 等 ${ }^{[22]}$ 和 Stedmon 等 ${ }^{[13]}$ 则发现 CDOM 在 $375 \mathrm{~nm}$ 处吸收系数 $a(375)$ 与 $S$ 值存在显著的负相关. 从本 次研究结果来看, 比吸收系数与 $S$ 值也存在显著的线性负相关 (图 3), 而吸收系数与 $S$ 值则没关系. 在 $280-400 \mathrm{~nm}$ 波段比吸收系数与 $S 2$ 线性拟合的相关性最好，随着波长增加与 $S 2$ 的拟合度下降，而与 $S 3$ 的 拟合度则上升, 分析原因主要与 $S$ 值拟合的波段有关, 一般而言各波长处比吸收系数与其附近波段拟合得 到 $S$ 值相关性最好. $a(250) / a(365)$ 值也是表征 CDOM 组成的重要参数, 其与 $S$ 值存在显著的正相关关 系, 表示 CDOM 分子量越小, $S$ 值就越高. 表征 CDOM 组成的 3 个参数比吸收系数、 $a(250) / a(365)$ 值、 $S$ 值的关系大致是: 比吸收系数越大, $a(250) / a(365)$ 值和 $S$ 值就越小, 对应的 CDOM 分子量就越大, 其组 成中腐质酸的比例越高.

\section{4 CDOM 吸收与荧光关系}

CDOM 是一类苂光物质, 在紫外短波光的激发下会发出长于吸收光波长的苂光, 因而 CDOM 吸收既 可以直接用分光光度计测量得到，也可通过苂光值反演推算得到. 由于苂光的高灵敏性，加之现在有很多 野外现场观测的苂光仪, 因而人们纷纷利用苂光特性来研究 CDOM 浓度、时空分布甚至组成, 尤其是对吸 收系数非常低的寡营养水体. 但由于并不是 CDOM 中的所有物质在短波光的激发下都会发出苂光, 因而 在利用菼光来反演水体 CDOM 浓度时首先必须建立本区域苂光强度与吸收系数之间的定量线性关系. 在 近海、海湾、河口区等, 不同的研究者均建立起适合本地区的经验反演模型 ${ }^{[1,12,30]}$.

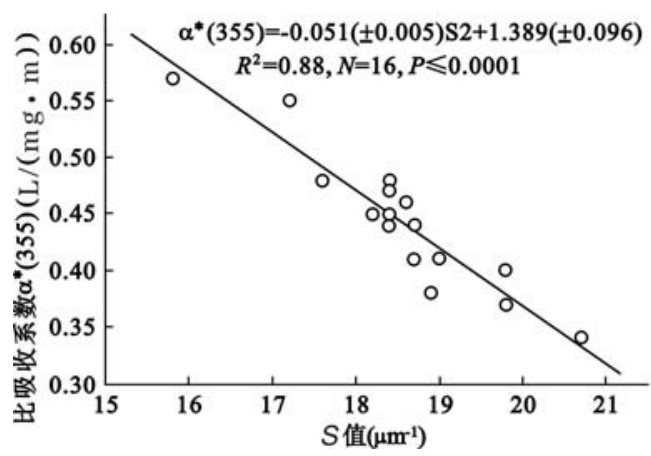

图 3 指数函数斜率 $S$ 值与比吸收系数线性拟合

Fig. 3 Linear regression of $S$ values and $a^{*}$ (355)

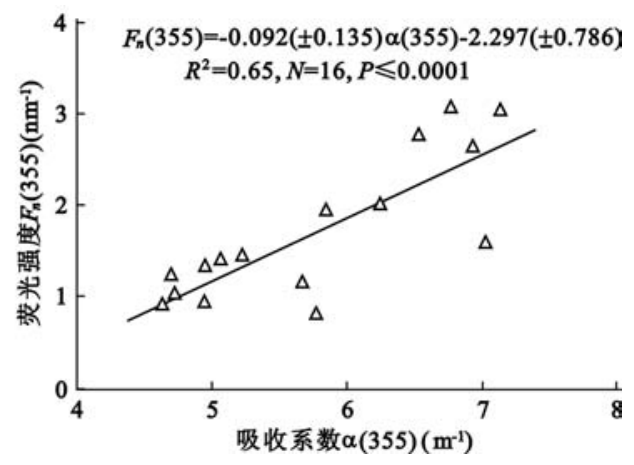

图 4 苂光强度与吸收系数线性相关

Fig. 4 Linear regression of $a(355)$ and $F_{n}(355)$

另外, 夏达英等 ${ }^{[31]}$ 对青岛近岸水黄色物质的菼光特性进行研究发现其激发峰值波长在 $340 \mathrm{~nm}$ 附近, 苂光光谱峰值波长在 $440 \mathrm{~nm}$ 附近. 但鉴于国际上一般采取 $355 \mathrm{~nm}$ 作为激发波长, $450 \mathrm{~nm}$ 作为发射波长, 为了比较起见, 我们选取 $355 \mathrm{~nm}$ 作为激发波长, $450 \mathrm{~nm}$ 作为发射波长. 菼光值的变化范围 $0.79-3.04$ $\mathrm{nm}^{-1}$ (平均值 $1.69 \pm 0.77 \mathrm{~nm}^{-1}$ ) (表 1 ), 高值基本上分布在河流人湖口、湾内及沿岸带, 低值则分布在湾 口地区, 如湾内的 M1、M7 - M11 的值均高于其他各点, 菼光最高值出现在梁溪河入湖口的 M1 点, 为 3.04 $\mathrm{nm}^{-1}$, 与 DOC 最高值分布一致. 由于高的荧光值对应着高的 CDOM 浓度, 因而苂光值的差异也进一步反 映了 CDOM 的空间分布, 这种分布主要是由于河流携带大量陆源 CDOM 人湖, 从湾内到湾口随着湖水的 不断稀释, CDOM 浓度不断下降, 对应的苂光值也逐渐降低. 国际上大多研究表明 ${ }^{[11,12,32]}$, 即便 CDOM 来源和化学组成差异万千, CDOM 吸收与荧光强度均存在显著线性关系. 本次研究也发现 CDOM 吸收系 数与其苂光值存在显著正相关 (图 4), CDOM 吸收与荧光强度的显著正相关结论进一步证实用苂光来反 演 CDOM 浓度的可行性, 为利用荧光来进行 CDOM 的水色遥感提供了坚实的理论基础. 


\section{3 结语}

本研究报道了太湖梅梁湾 CDOM 的空间分布, 呈现从河口往湾内、湾口递减的趋势, 建立的 CDOM 的 吸收光谱模型及苂光强度、DOC 浓度与 CDOM 吸收系数的线性相关式、各参数之间内在关系将有助于了解 CDOM 的组成及地球化学循环, 并为太湖水质遥感模型的建立做出一定贡献. 但关于太湖 CDOM 的研究 还有待进一步加强, 尤其是随着全球 UV-B 辐射的增强, 其引起水中 CDOM 光化学降解, 使大量大分子有 机物分解为容易被微生物利用的结构和分子量较小的有机物，势必会增强微生物的活性. 但另一方面， UV-B 辐射增强致使 CDOM 发生光降解又会带来 UV-B 辐射穿透能力的增加, 使得湖泊生物暴露在强的 UV-B 辐射下, 并且这种趋势会随着全球气候变化与酸雨问题的影响而得到增强. 此外, CDOM 的光降解 使得其代谢和分解产物 CO 浓度增加, 加剧全球的温室效应. 由此可见，湖泊水体中 CDOM 浓度及光降解 行为将影响到湖泊生态系统基本生命物质的生物地球化学进程.

\section{4 参考文献}

[1] Rochelle-Newall E J, Fisher T R. Chromophoric dissolved organic matter and dissolved organic carbon in Chesapeake Bay. Marine Chemistry, 2002, 77(1): $23-41$.

[2] Kirk J T O. Light and photosynthesis in aquatic ecosystem. Cambridge: Cambridge University Press. 1994.

[3] Williamson C E, Stemberger R S, Morris D P, et al. Ultraviolet radiation in North American lakes: Attenuation estimates from DOC measurements and implications for plankton communities. Limnol \& Oceanogr, $1996,41(5): 1024-1034$. .

[4] Morris, D P, Hargreaves B P. The role of photochemical degradation of dissolved organic carbon in regulating the UV transparency of three lakes on the Pocono Plateau. Limnol \& Oceanogr, 1997, 42 (2) : 239 -249 .

[5] Laurion I, Ventura M, Catalan J, et al. Attenuation of ultraviolet radiation in mountain lakes: Factors controlling the among- and within-lake variability. Limnol \& Oceanogr, 2000, 45(6) : $1274-1288$.

[6] Huovinen P S, Penttol H, Soimasuo M R. Spectral attenuation of solar ultraviolet radiation in humic lakes in Central Finland. Chemosphere, 2003, 51(3) : 205 - 214.

[7] Carder K L, Steward R G, Harvey G R, et al. Marine humic and fulvic acids: Their effects on remote sensing of ocean chlorophyll. Limnol \& Oceanogr, 1989, 34(1): 68 -81.

[8] Nelson N B, Siegel D A, Michaels A F. Seasonal dynamics of colored dissolved organic material in the Sargasso Sea. Deep-Sea Research I, 1998, 45(6) : 931 - 957.

[9] Rochelle-Newall E J, Fisher T R. Production of chromophoric dissolved organic matter fluorescence in marine and estuarine environments: an investigation into the role of phytoplankton. Marine Chemistry, 2002, $77(1): 7-21$.

[10] Bricaud A, Morel A, Prieur L. Absorption by dissolved organic matter of the sea (yellow substance) in the UV and visible domain. Limnol \& Oceanogr, 1981, 26(1): 43 - 53.

[11] Seritti A, Russo D, Nannicini L, et al. DOC, absorption and fluorescence properties of estuarine and coastal waters of the Northern Tyrrhenian Sea. Chemical Speciation and Bioavailability, 1998, 10(3) : 95 - 106.

[12] Ferrari G M, Dowell M D. CDOM Absorption Characteristics with relation to fluorescence and salinity in coastal areas of the Southern Baltic Sea. Estuarine, Coastal and Shelf Science, 1998, 47(1) : 91 - 105.

[13] Stedmon C A, Markager S, Kaas H. Optical Properties and Signatures of chromophoric dissolved organic matter (CDOM) in Danish coastal waters. Estuarine, Coastal and Shelf Science, 2000, 51(2) : 267 - 278.

[14] 吴永森, 张士鬼, 张绪琴等. 海水黄色物质光吸收特性实验研究. 海洋与湖沼, 2002, 33 (4) : 402 -406 .

[15] 陈楚群, 潘志林, 施 平. 海水光谱模拟及其在黄色物质遥感反演中的应用. 热带海洋学报, 2003 , 
$22(5): 33-39$.

[16] 朱建华, 李铜基. 黄东海非色素颗粒与黄色物质的吸收系数光谱模型研究. 海洋技术, 2004, 23(2): $7-13$.

[17] 杨顶田,陈伟民, 吴生才等. 湖泊中有色可溶性物质对近紫外及蓝光衰减的影响. 湖泊科学, 2003, 15 (3) : $269-274$.

[18] 张运林, 秦伯强, 马荣华等. 太湖典型草、藻型湖区有色可溶性有机物的吸收及苂光特性. 环境科学, $2005,26(2)$ : $142-147$.

[19] 张运林, 吴生才, 秦伯强等. 太湖梅梁湾有色可溶性有机物对光的吸收. 中国环境科学, 2004,24 (4) : $405-409$.

[20] 马荣华, 戴锦芳, 张运林. 东太湖 CDOM 吸收光谱的影响因素与参数确定. 湖泊科学, 2005, 17(2): $120-126$.

[21] Keith D J, Yoder J A, Freeman S A. Spatial and temporal distribution of coloured dissolved organic matter (CDOM) in Narragansett Bay, Rhode Island: implications for phytoplankton in coastal waters. Estuarine, Coastal and Shelf Science, 2002, 55(5): $705-717$.

[22] Del Castillo C E, Coble P G. Seasonal variability of the colored dissolved organic matter during the $1994-$ $95 \mathrm{NE}$ and SW Monsoons in the Arabian Sea. Deep-Sea Research II, 2000, 47: 1563 - 1579.

[23] De Haan H. Solar UV-light penetration and photodegradation of humic substances in peaty lake water. Limnolo \& Oceanogr, 1993, 38(5) : 1072 - 1076.

[24] Frenette J J, Arts M. T, Morin J. Spectral gradients of downwelling light in a fluvial lake (Lake Saint-Pierre, St-Lawrence River). Aquatic Ecology, 2003, 37(1) : 77 - 85.

[25] Morris D P, Zagarese H, Williamson C E, et al. The attenuation of solar UV radiation in lakes and the role of dissolved organic carbon. Limnolo \& Oceanogr, 1995, 40(8) : $1381-1391$.

[26] De Lange H J. The attenuation of ultraviolet and visible radiation in Dutch inland waters. Aquatic Ecology, $2000,34(3): 215-226$.

[27] Gallie E A. Variation in the specific absorption of dissolved organic carbon in Northern Ontario lakes. In: Ocean Optics XIII, Steven G A, Robert F, eds. Proc. SPIE 2963, 1997: 417 - 422.

[28] Yacobi Y Z, Alberts J J, Takács M, et al. Absorption spectroscopy of colored dissolved organic carbon in Georgia (USA) rivers: the impact of molecular size distribution. Journal of Limnology, 2003, 62 (1): 41 -46 .

[29] Markager W, Vincent W F. Spectral light attenuation and absorption of UV and blue light in natural waters. Limnol \& Oceanogr, 2000, 45(3): $642-650$.

[30] Kowalczuk P, Cooper W J, Whitehead R F, et al. Characterization of CDOM in an organic-rich river and surrounding coastal ocean in the South Atlantic Bight. Aquatic Science, 2003, 65(4) : $384-401$.

[31] 夏达英, 李宝华, 吴永森等. 海水黄色物质苂光特性的初步研究. 海洋与湖沼, 1999, 30(6) : 719 -725 .

[32] Hoge F E, Vodacek A, Blough N V. Inherent optical properties of the ocean: retrieval of the absorption coefficient of chromophoric dissolved organic matter from fluorescence measurements. Limnol \& Oceanogr, $1993,38(7): 1394-1402$. 